\title{
Advancing Lidar Sensors Technologies for Next Generation Landing Missions
}

\author{
Farzin Amzajerdian ${ }^{1}$, Glenn D. Hines ${ }^{2}$, Vincent E. Roback ${ }^{3}$, Larry B. Petway ${ }^{1}$, Bruce W. Barnes ${ }^{1}$, Paul F. \\ Brewster ${ }^{4}$ \\ NASA Langley Research Center, Hampton, Virginia, 23681
}

Diego F. Pierrottet

Coherent Applications, Inc., Hampton, Virginia, 23666

and

Alexander Bulyshev

Analytical Mechanics Associates, Hampton, VA 23666

\begin{abstract}
Missions to solar systems bodies must meet increasingly ambitious objectives requiring highly reliable "precision landing", and "hazard avoidance" capabilities. Robotic missions to the Moon and Mars demand landing at pre-designated sites of high scientific value near hazardous terrain features, such as escarpments, craters, slopes, and rocks. Missions aimed at paving the path for colonization of the Moon and human landing on Mars need to execute onboard hazard detection and precision maneuvering to ensure safe landing near previously deployed assets. Asteroid missions require precision rendezvous, identification of the landing or sampling site location, and navigation to the highly dynamic object that may be tumbling at a fast rate. To meet these needs, NASA Langley Research Center (LaRC) has developed a set of advanced lidar sensors under the Autonomous Landing and Hazard Avoidance Technology (ALHAT) project. These lidar sensors can provide precision measurement of vehicle relative proximity, velocity, and orientation, and high resolution elevation maps of the surface during the descent to the targeted body. Recent flights onboard Morpheus free-flyer vehicle have demonstrated the viability of ALHAT lidar sensors for future landing missions to solar system bodies.
\end{abstract}

\section{Introduction}

Over the past decade, NASA has been actively pursuing lidar sensor technologies aimed at enabling precision navigation and terrain hazard avoidance for future missions to solar system bodies including robotic and human missions to the Moon, Mars, and asteroids. Robotic missions to the Moon and Mars demand landing at pre-designated sites of high scientific value near hazardous terrain features, such as escarpments, craters, slopes, and rocks. Missions planned for paving the path to colonization of the Moon and human landing on Mars need to execute onboard hazard detection and precision maneuvering to ensure safe landing near previously deployed assets. Asteroid missions require precision rendezvous, identification of the landing or sampling site location, and navigation to the highly dynamic object that may be tumbling at a fast rate. Lidar technology will play a major role in enabling these ambitious missions due to its capabilities of providing precision measurement of vehicle relative proximity and velocity, and providing high resolution elevation maps of the surface during the approach to the targeted body. Other space-related applications of these lidars are automatic spacecraft rendezvous and docking, satellite servicing, and space debris identification and removal. To demonstrate the capabilities of lidar sensors, NASA Langley Research Center (LaRC)

\footnotetext{
${ }^{1}$ Laser Remote Sensing Branch, NASA Langley Research Center, Mail Stop 468.

2 Systems Engineering \& Engineering Methods Branch, NASA Langley Research Center, Mail Stop 131.

${ }^{3}$ Remote Sensing Flight Systems Branch, NASA Langley Research Center, Mail Stop 468.

${ }^{4}$ Flight Software Systems Branch, NASA Langley Research Center, Mail Stop 472.
} 
has developed a set of advanced lidar sensors under the Autonomous Landing and Hazard Avoidance Technology (ALHAT) project ${ }^{1}$.

These lidar sensors are: a 3-Dimensional Imaging Flash Lidar, a Laser Altimeter, and a Doppler Lidar. The Flash Lidar is capable of generating elevation maps of the terrain to indicate hazardous features such as rocks, craters, and steep slopes. It generates $16 \mathrm{k}$ pixels range images with $8 \mathrm{~cm}$ precision, at $20 \mathrm{~Hz}$ frame rate, from a maximum slant range of $1800 \mathrm{~m}$ to about $100 \mathrm{~m}$ from the target area. The Laser Altimeter can provide long range altitude from over $50 \mathrm{~km}$ with $10 \mathrm{~cm}$ precision for improving the vehicle position data from the Inertial Measurement Unit. The Doppler Lidar provides ground velocity vector and altitude data from a few kilometers altitude, to about $1 \mathrm{~cm} / \mathrm{sec}$ and $20 \mathrm{~cm}$ precision respectively, for improving the vehicle state knowledge, triggering critical descent and landing sequence events, and executing precision divert maneuvers. Over the course of their development from concept to prototype units, these sensors were subjected to extensive static and dynamic tests at the NASA-LaRC lidar test range, and from helicopter and fixed-wing aircraft platforms. The scope of each test expanded as the lidar systems development progressed in order to show their operation as an integrated sensor with other ALHAT landing system components, namely the Hazard Detection System (HDS) ${ }^{2}$, developed by NASA Jet Propulsion Laboratory (JPL) and the Autonomous Guidance, Navigation, and Control (AGN\&C) system developed by NASA Johnson Space Center (JSC) and Charles Stark Draper Laboratory (CSDL) ${ }^{3}$. The ALHAT Landing system was then integrated onto Morpheus ${ }^{4}$ vehicle, a rocket-powered terrestrial flight-test platform built by NASA Johnson Space Center (JSC) to fully demonstrate the hazard avoidance and precision landing capabilities. A total of five flights were conducted in spring of 2014 at NASA Kennedy Space Center where we had constructed a simulated lunar terrain site consisting of realistic hazard features and designated landing areas ${ }^{5}$. The lidar sensors operated nominally in all five lights, providing consistent and accurate 3-D terrain maps and navigation data. Two additional flights are planned in December 2014 that will provide more information on operation of lidar sensors as integrated component and landing system and help improving the utilization of their data by hazard avoidance and navigation algorithms. The Morpheus flight tests mark a major milestone for the lidar sensors on a roadmap to the development of space flight units for future missions to solar system bodies. The next phase of the roadmap is maturation of the sensors that consists of design optimization and space qualification of their components.

\section{Description of Lidar Sensors}

The development of the lidar sensors was initiated about eight years ago from design concepts to operational prototype systems. The operational concept of each of the lidar sensors are explained below and the performance and capabilities of each of the prototype units demonstrated by Morpheus flights are described.

\section{A. Navigation Doppler Lidar}

The Navigation Doppler Lidar (NDL) utilizes a frequency modulated continuous wave (FMCW) technique to obtain high-resolution range and velocity information. The principle of the lidar operation is illustrated in Fig. 1 showing the modulation waveform consisting of three segments: up-ramp chirp, constant frequency, and down-ramp chirp. The resultant returned waveform from the target is delayed by $t_{d}$, the light round trip time. When the target or the lidar platform is not stationary during the beam round trip time, the signal frequency will be also shifted up or down, depending on the velocity direction, due to the Doppler effect. When mixing the two waveforms at the detector, an interference signal is generated whose frequency is equal to the difference between the transmitted and received frequencies. In absence of velocity along the laser beam, the signal frequency during the "up-ramp" and "down-ramp" periods are equal and their magnitude is directly proportional to the distance to the target. When the vehicle is moving, the up-ramp and down-ramp frequencies will not be equal and their difference is related to the Doppler velocity. The target range and magnitude of the velocity component along the laser beam are determined through the following simple equations:

$$
R=\left(\frac{T C}{2 B}\right)\left(\frac{f_{I F}^{+}-f_{I F}^{-}}{2}\right) \quad V=\left(\frac{\lambda}{2}\right)\left(\frac{f_{I F}^{+}+f_{I F}^{-}}{2}\right)
$$

where $f_{I F}^{+}$and $f_{I F}^{-}$are the intermediate up-ramp and down-ramp frequencies, $B$ is the modulation bandwidth, $T$ is the waveform period, $C$ is the speed of light, and $\lambda$ is the laser wavelength.

The constant frequency segment also produces the Doppler velocity that can be used for eliminating the data dropouts when either up-ramp or down-ramp frequency is very close to zero and allows for eliminating the measurement ambiguities that may arise in certain scenarios. 


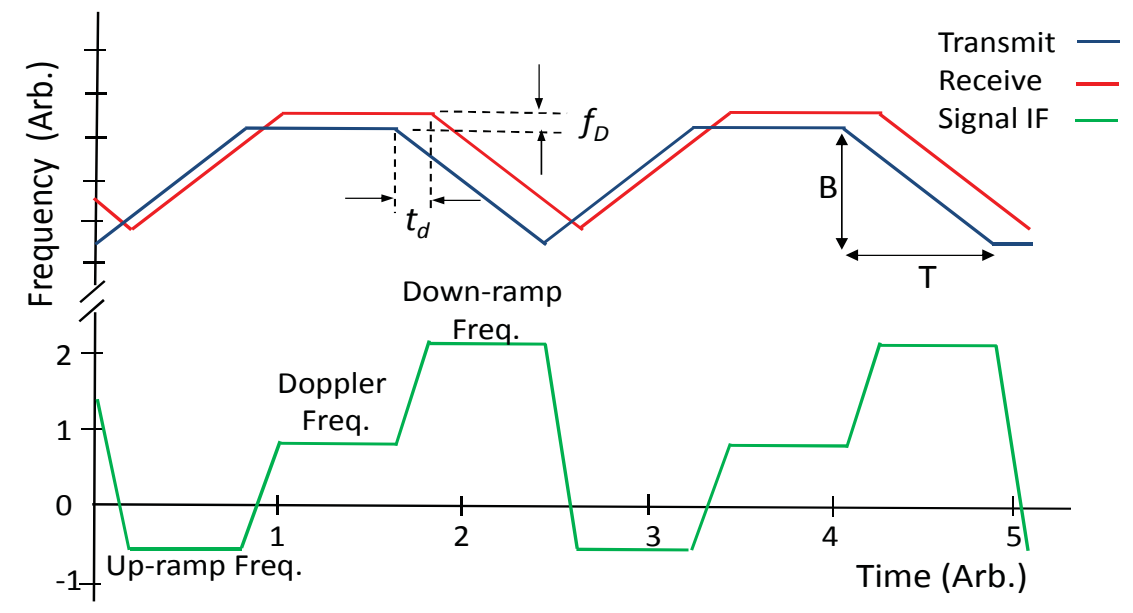

Figure 1. Linearly frequency modulated transmitted beam and returned signal, and the resulting intermediate frequency (IF) of the homodyne signal.

Figure 2 illustrates the system design utilizing an optical homodyne configuration. A relatively low power, single frequency laser operating at eye safe wavelength of 1.55 micron, is used as the master oscillator. The output of this laser is modulated per the waveform of Fig. 1. Part of the laser output is amplified to be transmitted and the remaining is used as the local oscillator (LO) for optical homodyne detection. The lidar transmits three laser beams in different directions and the returns are directed to three corresponding receivers.

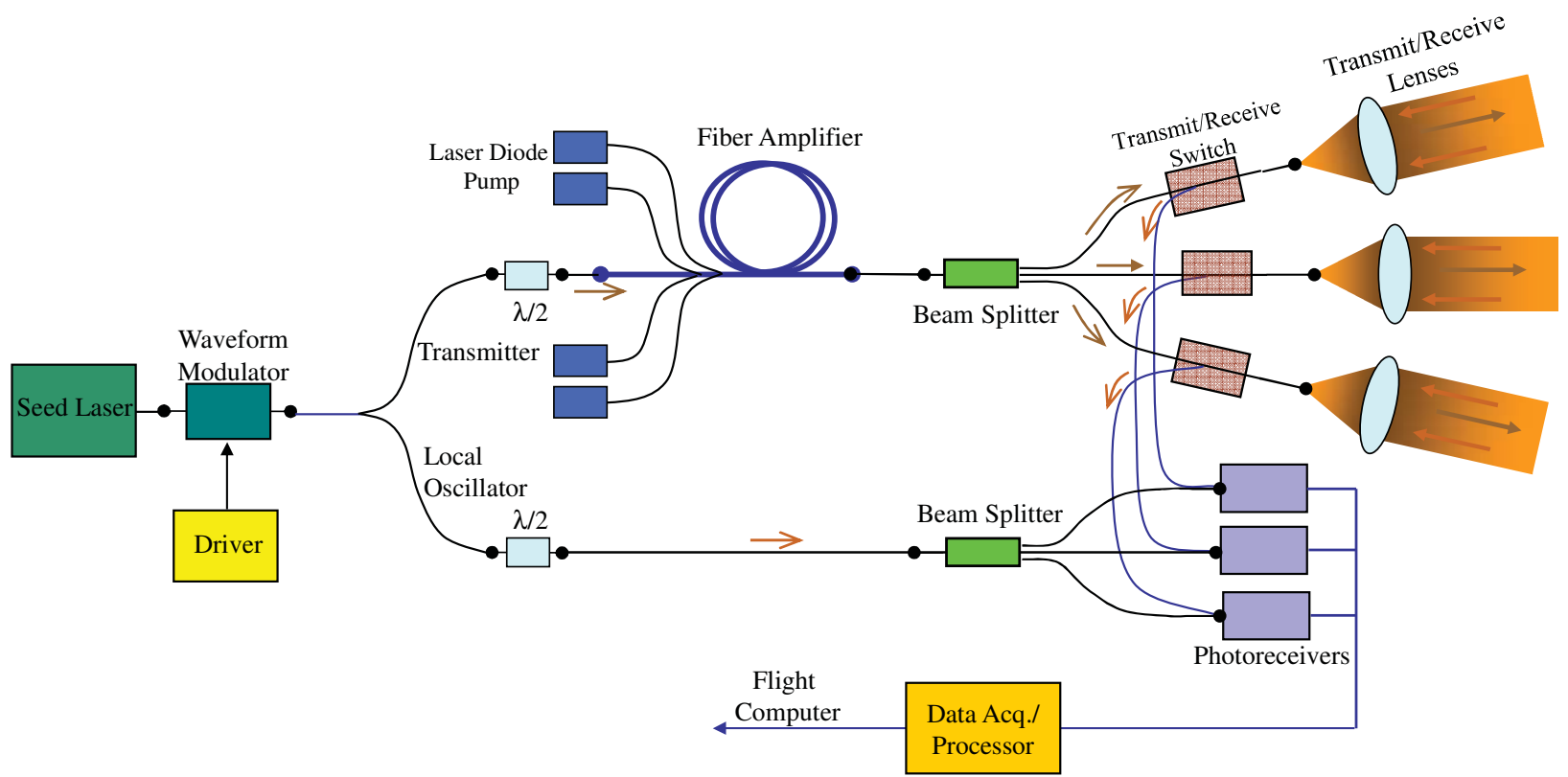

Figure 2. Doppler lidar system configuration illustrating three transmitted beams and their corresponding receivers providing line-of-sight velocity and range measurements in three different directions.

A single board computer (SBC) is used as the Command and Data Handling (C\&DH) system which responds to commands from the vehicle, transmits the instrument's measurements, interfaces to the real-time processor, and controls and monitors the internal components of the lidar. The Doppler lidar signal processor digitizes and processes the output of three optical receivers in real-time. A functional block diagram of the lidar signal processor is shown in Fig. 3. The primary electronic data processing component of the Doppler lidar is a field programmable gate array (FPGA) board. The digitized outputs of the three analog receivers are de-multiplexed in the time domain to separate the up-ramp, constant (Doppler), and down-ramp portions of the signal. The FPGA then applies a set of high-resolution fast Fourier transforms (FFT) to each segment of the waveform for each of the three beams. From these measured 
frequencies, the LOS velocity and range data are determined. The platform vector velocity is obtained from the LOS velocities measured along the three laser beams by using a priori pointing knowledge. Using all three LOS range measurements allows determination of the vehicle altitude relative to the local ground without the need for vehicle attitude angle data from a separate sensor. The use of three beams also reduces the effect of terrain features such as boulders and craters on the altitude data. Additionally, the vehicle attitude relative to the ground can be derived from the three LOS range measurements.

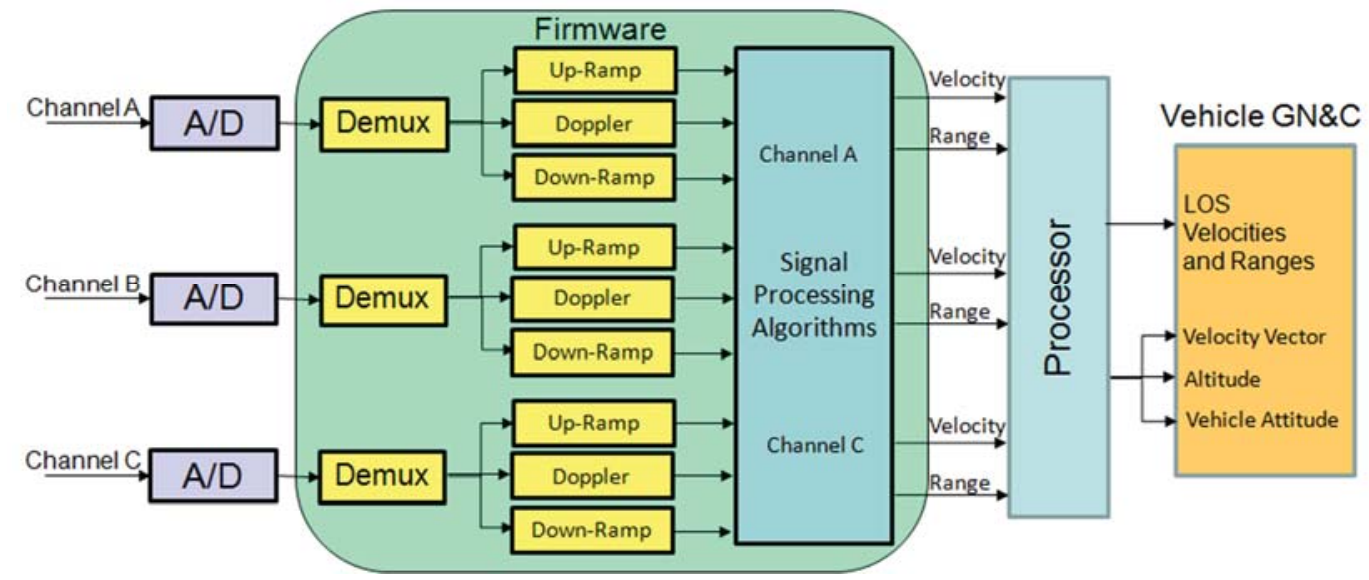

Figure 3. Real-time signal processor functional diagram.

Figure 4 shows the prototype Doppler lidar consisting of an electronics chassis and an optical head that houses three fiber-coupled lenses. The optical head is mounted rigidly on the vehicle with a clear field-of-view to the ground, and connected to the electronic chassis through a long fiber optic cable carrying the transmitted beams to the lenses and directing the collected signals from the same lenses to the receivers. The electronic chassis contains all the lidar components, including transmitter laser, receiver, real-time processor, system controller, and power supply unit. The prototype lidar was subjected to environmental tests to validate its construction workmanship and to ensure its operation on different test vehicles. The specifications of the prototype Doppler lidar are summarized in Table 1.

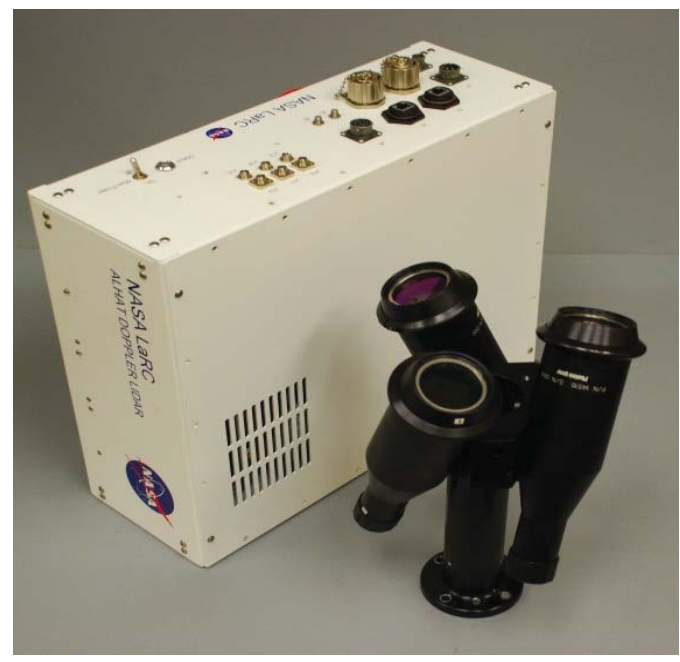

Table 1. Doppler Lidar Prototype System Specifications.

\begin{tabular}{|l|l|}
\hline Parameter & Prototype Lidar \\
\hline Line-Of-Sight Velocity Resolution & $0.2 \mathrm{~cm} / \mathrm{sec}$ \\
\hline Line-Of-Sight Range Resolution & $17 \mathrm{~cm}$ \\
\hline Attitude Resolution & $0.4 \mathrm{deg}$ \\
\hline Maximum Operational Altitude & $2200 \mathrm{~m}$ \\
\hline Data Rate & $20 \mathrm{~Hz}$ \\
\hline
\end{tabular}

Figure 4. Prototype Doppler lidar system consisting of an electronic chassis and an optical head

\section{B. 3-Dimensional Imaging Flash Lidar}

The Flash Lidar is capable of generating three dimensional (3D) images of the terrain at video rates with sufficient lateral resolution and range precision for detecting hazardous features $30 \mathrm{~cm}$ or greater in size from almost $2 \mathrm{~km}$ slant range. The principal of Flash Lidar is illustrated in Fig. 5. The Flash Lidar uses a two dimensional detector array to detect a laser pulse return from the target. The detector's Readout Integrate Circuit (ROIC) measures the laser pulse time of arrival of each individual pixel simultaneously. Thus each flash of the laser generates a 3D image of the target 
illuminated by the laser beam. In older, more conventional imaging lidar systems, the laser beam is scanned over the targeted area in a raster pattern and a single detector is used to detect consecutive pulses. Thus many laser pulses are required to cover the target area and generate a multi-pixel image with sufficient resolution. The major challenge with such a scanning system is controlling the laser beam pointing from a moving platform and then estimating the laser spot position in the target area for each transmitted pulse. By recording a full 3D image with a single laser pulse, the Flash Lidar provides a much higher image frame rate, eliminates the need for a fast laser beam scanning mechanism, and mitigates the effects of platform motion.

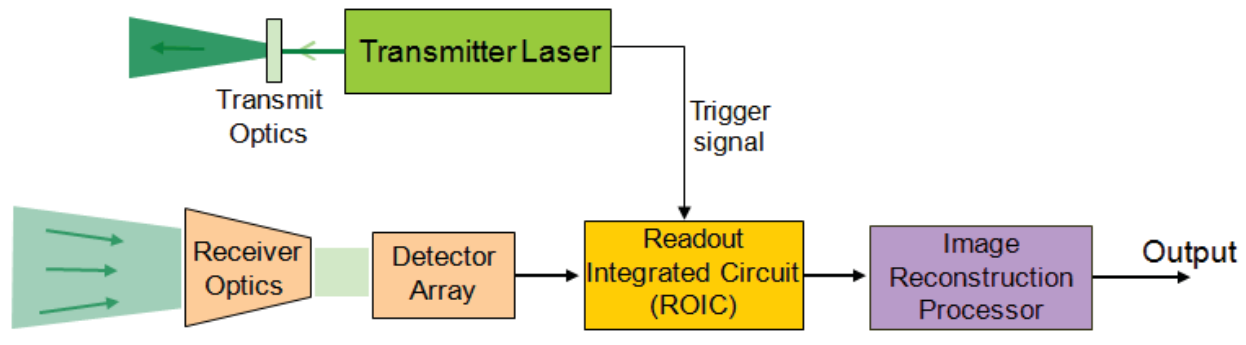

Figure 5. Schematic of Flash Lidar sensor system.

The Flash Lidar developed for ALHAT uses a 3D imaging camera, also referred to as the sensor engine, developed by Advanced Scientific Concepts (ASC). ${ }^{6,7}$ The sensor engine consists of a detector array integrated with a matching ROIC packaged with the associated detector/ROIC power supply and control electronics and a real-time processor that calibrates the output of the ROIC and outputs both range and intensity image frames. The ASC sensor engine has a 128x128 pixel array that is capable of generating real-time images at up to 30 frames per second. Fig. 6 shows the prototype Flash Lidar consisting of a sensor head and an electronics chassis. The sensor head houses the sensor engine, the transmitter laser, and the transmit/receive optics, while the electronics box houses the sensor Controller and Data Handling $(\mathrm{C} \& \mathrm{DH})$ unit, the laser driver, the power conditioning/distribution unit, and temperature control boards. The transmitter laser, developed by Fibertek, operates at 1.06 micron wavelength generates a uniform square shape beam, matching the detector array, with a divergence adjusted to match the receiver field of view. The lidar C\&DH unit performs a number of functions including controlling and monitoring various lidar components, interfacing with avionics, and performing image processing and conditioning. The C\&DH executes a median filter algorithm for eliminating sporadic range noise, masks the known bad pixels, and applies an absolute range calibration routine. The Flash Lidar system and performance specifications are summarized in Table 2. The Flash Lidar is able to detect hazardous terrain features as small as $30 \mathrm{~cm}$ from over $1 \mathrm{~km}$ distance. The maximum operational distance of the Flash Lidar can be extended by reducing the divergence of its transmitter laser and illuminating a subset of its detector pixels. Assuming no atmospheric attenuation, the maximum operational range of the lidar is inversely proportional to the number of illuminated pixel squared. For example, the lidar's operational range increases from $1800 \mathrm{~m}$ to $20 \mathrm{~km}$ by reducing the number of illuminated pixels from 128x128 to 11x11. Therefore, the Flash Lidar can provide useful low resolution 3-D images of the terrain and altitude data even during the initial descent phase.
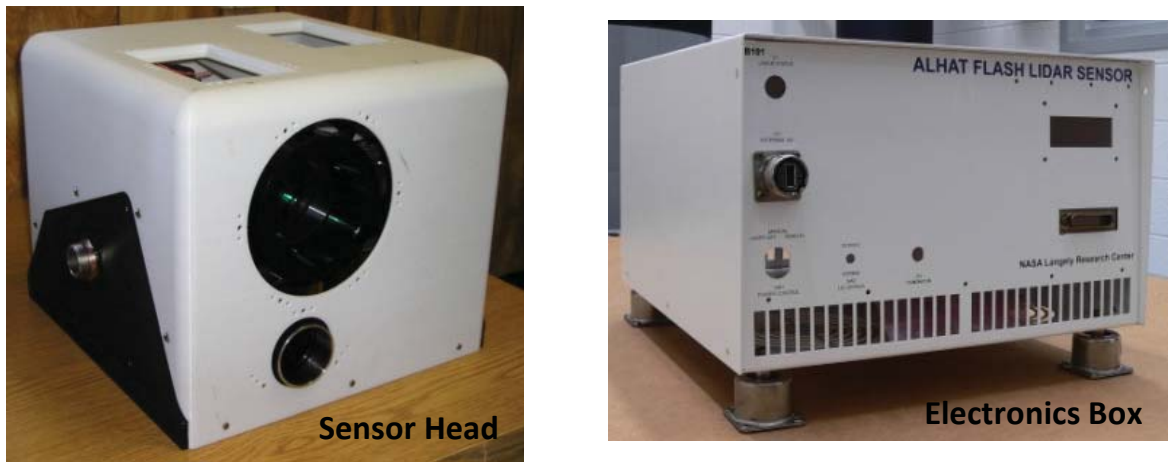

Figure 6. Prototype Flash Lidar sensor. 
Table 2. Specifications of the Flash Lidar Prototype System.

\begin{tabular}{|l|l|}
\hline Parameter & Value \\
\hline Arrav Size & $128 \mathrm{X} 128$ \\
\hline Operational Wavelength & $1.06 \mathrm{micron}$ \\
\hline Laser Pulse energy & $50 \mathrm{~mJ}$ \\
\hline Laser Pulsewidth & $8 \mathrm{nsec}$ \\
\hline Receiver Field of View & $1 \mathrm{deg}$. and $5 \mathrm{deg}$ \\
\hline Receiver Aperture Diameter & $100 \mathrm{~mm}$ \\
\hline Frame Rate & $20 \mathrm{~Hz}$ \\
\hline $\begin{array}{l}\text { Max operational range (natural } \\
\text { terrain target) }\end{array}$ & $1800 \mathrm{~m} @ 90^{\circ}$ look angle \\
\cline { 2 - 2 } & $950 \mathrm{~m} @ 30^{\circ}$ look angle \\
\hline Range Precision & $8 \mathrm{~cm}$ \\
\hline
\end{tabular}

\section{Laser Altimeter}

As noted earlier, the vehicle altitude can be measured by the Flash Lidar at high altitudes of over $20 \mathrm{~km}$ and the Doppler lidar from about $2 \mathrm{~km}$ above the ground. However, a separate Laser Altimeter sensor can ease the spacecraft design and provide redundancy to this critical data. Figure 7 shows the Laser Altimeter system diagram. The Laser Altimeter uses a moderate power laser operating at an eye safe energy level at 1.57 micron wavelength for easier terrestrial operation and testing. The laser generated a few nanosenonds long pulses that combined with high resolution Time-of-Arrival (TOF) counter allows for highly precise distance measurements. The prototype system, as shown in Fig. 8, is significantly more powerful and more precise than the ALHAT project required and it also exceeds the performance requirements of any foreseeable landing mission. Altitude measurements with $0.5 \mathrm{~m}$ precision from about $20 \mathrm{~km}$ is more than sufficient for mitigating the drift by the Inertial Measurement Unit (IMU) and providing an accurate vehicle position estimate. The measurements of the terrain contours required for Terrain Relative Navigation, as described in following section, requires similar operational range and precision specifications. The current sensor exceeds these performance requirements by an order of magnitude. Table 3 lists the performance specifications of the Laser Altimeter prototype.

Other important features of the Laser Altimeter are multi-return and automatic detection threshold capabilities. The Laser Altimeter allows for three returned pulses for each transmitted pulse to facilitate operation in presence of clouds. The automatic threshold enables reliable operation with a fixed low false alarm rate over its operational range ${ }^{8}$.

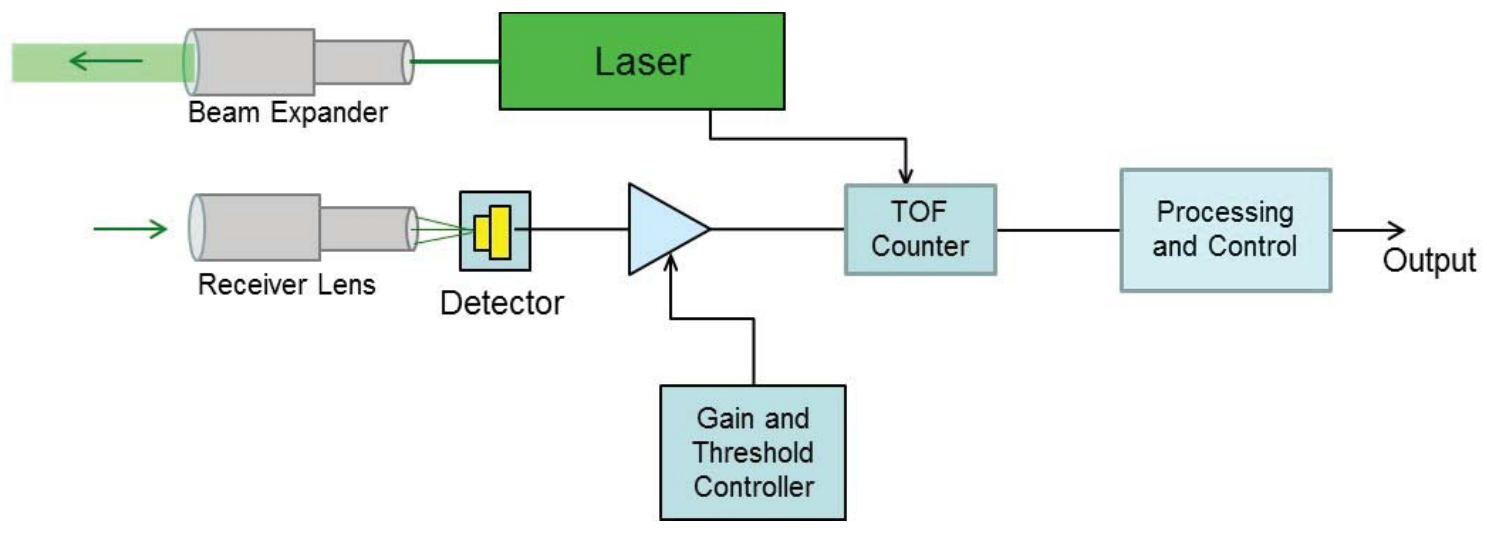

Figure 7. Laser Altimeter Sensor System Diagram. 


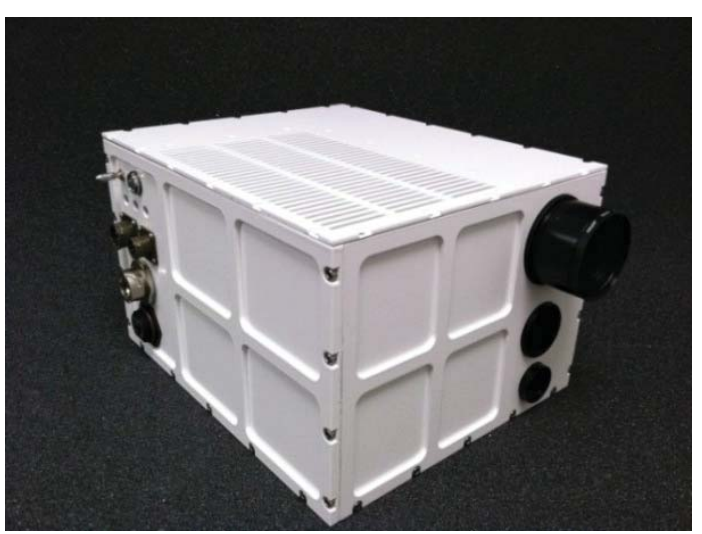

Table 3. Laser Altimeter Prototype System Specifications.

\begin{tabular}{|l|l|}
\hline Parameter & Prototype Lidar \\
\hline Max Operational Range & $50 \mathrm{~km}$ \\
\hline Accuracy & $0.0034 \%$ \\
\hline Resolution & $5 \mathrm{~cm}$ \\
\hline Data Update Rate & $30 \mathrm{~Hz}$ \\
\hline Operational Wavelength & 1.57 micron \\
\hline
\end{tabular}

Figure 8. Prototype Laser Altimeter sensor system.

\section{Operational Concept}

The three lidar sensors, described above, perform five essential functions during descent and landing phases: Altimetry, Velocimetry, Terrain Relative Navigation (TRN), Hazard Detection and Avoidance (HDA) and Hazard Relative Navigation (HRN). Table 4 below summarizes the functions that each lidar sensor is capable of performing along with their associated nominal operational altitudes during the descent, terminal approach, and landing phases for the Moon and Mars landings. There is a significant overlap in capabilities of these three lidar sensors. The Flash Lidar is capable of performing all the functions with exception of velocimetry, for which the Doppler Lidar provides. The ability of the Doppler Lidar to provide velocity data to better than $1 \mathrm{~cm} / \mathrm{sec}$ is highly attractive for precision landing. Additionally, the Doppler Lidar provides high resolution altitude and ground-relative attitude data that can further improve precision navigation to the identified landing site. The Laser Altimeter provides independent altitude data over a large operational altitude range from $50 \mathrm{~km}$ to $100 \mathrm{~m}$. Although the Flash lidar is fully capable of providing the necessary altitude data, the Laser Altimeter may present a more desirable option. The orientation of the spacecraft will be different during different phases of descent and landing, thus it may be difficult to accommodate the Flash Lidar such that it will have a clear view of the ground during the powered descent phase ( $20 \mathrm{~km}-5 \mathrm{~km}$ altitude), for performing Altimetry and TRN, as well as the approach phase ( $<2 \mathrm{~km}$ altitude), when it must perform HDA and HRN functions. Therefore, the Laser Altimeter can lessen the complexity of spacecraft accommodation design and ease the spacecraft attitude control requirements. Even if the Flash Lidar can operate over the entire powered descent phase, the Laser Altimeter may serve as a redundant sensor for either or both Altimetry and TRN functions.

Table 4. Landing lidar sensors and their functions.

\begin{tabular}{|l|l|c|c|}
\hline \multirow{2}{*}{ Sensor } & \multirow{2}{*}{ Function } & \multicolumn{2}{|c|}{ Nominal Operational Altitude Range } \\
\cline { 3 - 4 } & & Lunar Landing & Mars Landing \\
\hline \multirow{3}{*}{ Flash Lidar } & HDA/HRN & $1000 \mathrm{~m}-100 \mathrm{~m}$ & $1000 \mathrm{~m}-100 \mathrm{~m}$ \\
\cline { 2 - 4 } & TRN & $15 \mathrm{~km}-5 \mathrm{~km}$ & $10 \mathrm{~km}-3 \mathrm{~km}$ \\
\cline { 2 - 4 } & Altimetry & $20 \mathrm{~km}-100 \mathrm{~m}$ & $10 \mathrm{~km}-100 \mathrm{~m}$ \\
\hline \multirow{2}{*}{ Doppler Lidar } & Velocimetry $^{1}$ & $2000 \mathrm{~m}-20 \mathrm{~m}$ & $4000 \mathrm{~m}-5 \mathrm{~m}$ \\
\cline { 2 - 4 } & Altimetry & $2000 \mathrm{~m}-20 \mathrm{~m}$ & $4000 \mathrm{~m}-5 \mathrm{~m}$ \\
\hline \multirow{2}{*}{ Laser Altimeter } & Altimetry & $20 \mathrm{~km}-100 \mathrm{~m}$ & $50 \mathrm{~km}-100 \mathrm{~m}$ \\
\cline { 2 - 4 } & TRN $^{1}$ & $15 \mathrm{~km}-5 \mathrm{~km}$ & N.A. \\
\hline
\end{tabular}

${ }^{1}$ Secondary function, maybe considered as redundancy option.

The operational scenario described above represents just a nominal case and the selection of sensors and their operational specifications can vary greatly depending on the mission and its Entry, Descent, and Landing (EDL) system design. Some of the factors that greatly influence the architecture of the sensors suite and its operational characteristics are landing accuracy, landing site terrain features, and the vehicle size. A landing mission may only need a Flash Lidar to perform the TRN function to achieve its required landing accuracy while another may only need 
the Doppler Lidar for precision navigation during terminal descent phase and execution of a soft touchdown. Table 4 represents nominal operational scenarios for the Moon and Mars landing. The presence of atmosphere and a larger gravity force make the Mars landing very different from a Moon landing and thus a different set of requirements for the sensors. For example, an un-guided parachute descent with large swings and a protective heat-shield will restrict the operational periods of the lidar sensors that must have a clear view of the ground. Asteroid missions are yet very different from the Moon and Mars, as the primary objectives of the sensors are to generate elevation map of the object and measure the vehicle relative bearings in addition to precise proximity and velocity data.

\section{Current State of Technology}

The capabilities of the landing lidar sensors were assessed and their performance were characterized during different phases of their development through extensive testing at NASA-LaRC Lidar Test Range facility and five helicopter and fixed-wing aircraft flight test campaigns. The first four flight test campaigns provided invaluable data for the development of fully autonomous prototype systems. The prototype lidar sensors were engineered to be as compact and robust as possible within the project budget and schedule constraints. Upon their completion, the prototype lidars were integrated with the HDS and AGN\&C system and installed in an instrumented truck to conduct integration tests in a dynamic environment at the NASA-LaRC Lidar Test Range ${ }^{9,10}$. HDS uses the Flash Lidar images to generate a digital elevation map of the landing area and select the best landing location given the vehicle's constraints and the mission objectives. AGN\&C uses the velocity and altitude data from the Doppler Lidar and Laser Altimeter to precisely determine the vehicle position and navigate the vehicle to the safe landing site provided by HDS. The integration tests at NASA-LaRC Test Range verified the command and data interfaces between the sensors, HDS, and AGN\&C system, and helped the development of procedures for registering the pointing of lidar beams. The last helicopter test (field test 5 campaign) was aimed to test the complete ALHAT landing system with the prototype sensors operating in concert with the HDS and AGN\&C system prior to the closed-loop demonstration flights. Figure 9 shows the lidar sensors mounted beneath the helicopter. The prototype systems performed reliably during the twoweek flight test 5 campaign ${ }^{11,12}$.

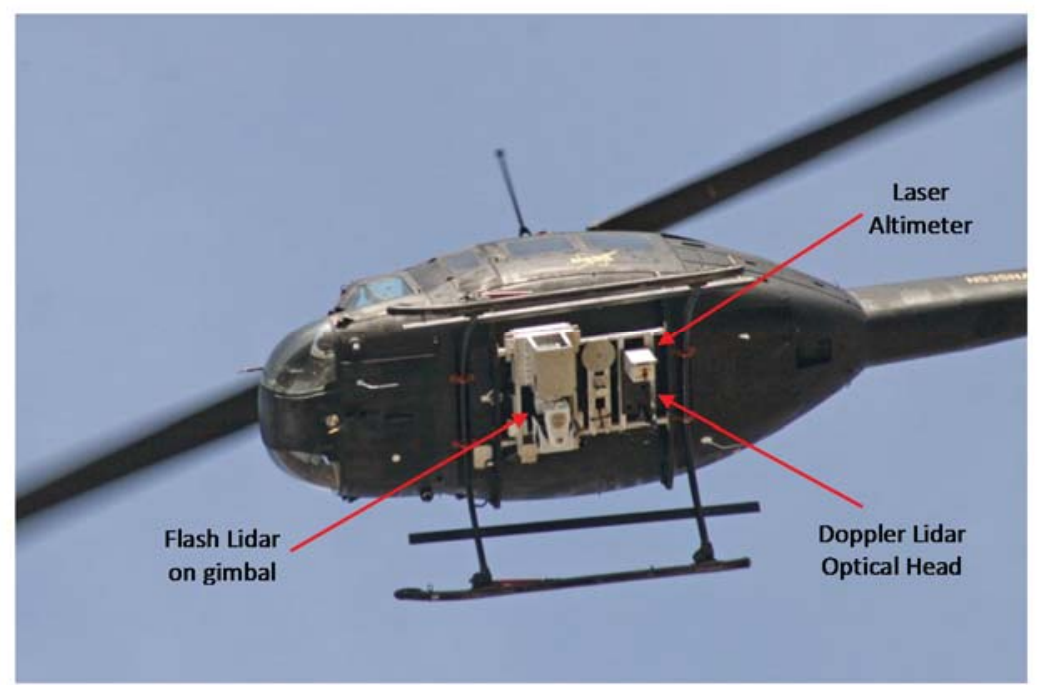

Figure 9. Prototype lidar sensors mounted under a Huey helicopter during Field Test 5. The Flash Lidar and Doppler Lidar electronic chassis were placed in a rack inside the cabin.

The prototype lidars were later integrated onto a rocket-powered, terrestrial flight-test vehicle (Fig. 10). The test vehicle, referred to as Morpheus ${ }^{8}$, was built by NASA Johnson Space Center (JSC) to demonstrate advanced propulsion and GN\&C technologies for future landing missions. A series of integration tests were conducted to validate sensor interfaces and operational procedures. These integration tests included three tethered tests during which the lidars were activated to communicate and provide data to various avionics systems while the Morpheus vehicle was suspended from a crane and executed a controlled flight procedure. All these integration tests were in preparation for a full landing demonstration at a hazard field specifically constructed for this purpose near the north end of Shuttle Landing Facility (SLF) runway at NASA Kennedy Space Center (Fig. 11). The hazard field is a $100 \mathrm{~m}$ 
x $100 \mathrm{~m}$ area simulating a challenging lunar terrain consisting of realistic hazard features (rock piles and craters) and designated landing areas. The flight profile as shown in Fig. 10 is designed to demonstrate the autonomous safe landing system, including the lidar sensors, that controls the vehicle flight trajectory to the selected safe site and executes a landing maneuver. Morpheus takes off from a launch pad next to the SLF runway and climbs to $250 \mathrm{~m}$ and then travels toward the hazard field about $500 \mathrm{~m}$ away. A few seconds after the vehicle begins its descend trajectory, the Flash Lidar maps the hazard field and provides it to HDS to identify the safe landing locations and selects the best one. Once the coordinates of the landing location is provided to the navigation system, the vehicle uses the Laser Altimeter and Doppler lidar to precisely navigate to the selected location within the hazard field.

A total of five flights were conducted in spring of 2014, three open-loop and two closed-loop flights. For the openloop flights, the lidars operate nominally and the ALHAT navigation system process the lidars data but did not influence the vehicle controlled by its own GN\&C system. In close-loop flights, the ALHAT navigation system, using the lidars data, defines the vehicle state and its flight trajectory to the designated landing location. These flights were declared successful in demonstrating autonomous safe landing by using only onboard sensors. Two additional closedloop flights are planned in December 2014 after implementing some improvements based on the data and lessons learned from earlier flights. One change is re-locating the Doppler lidar optical head from vicinity of the engine $(\sim$ $1 \mathrm{~m}$ ) to a further and higher location and point the beams forward in order to avoid the engine heat plume that caused some data dropouts.

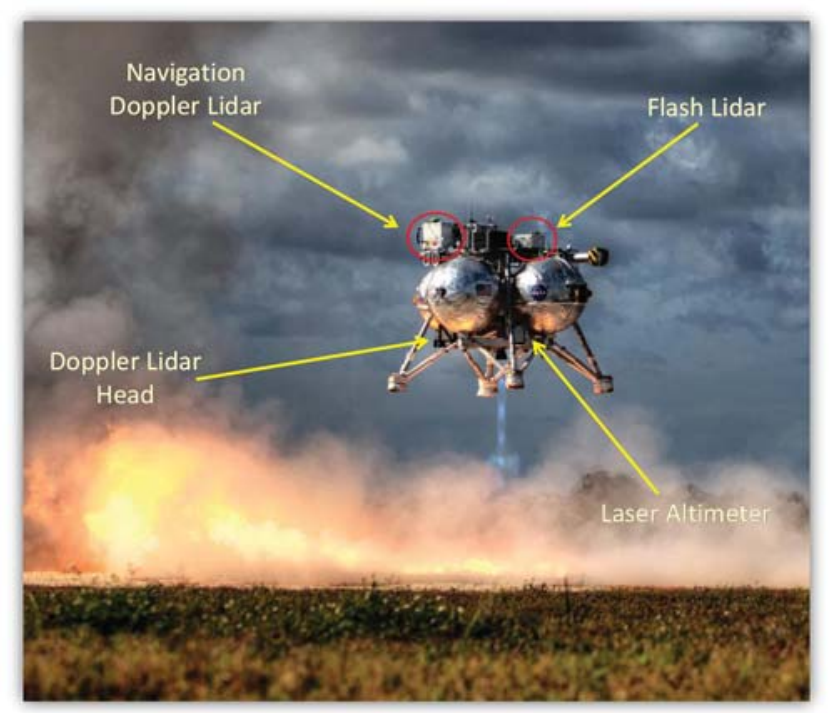

Figure 10. Lidar sensors integrated onto rocket-propelled Morpheus vehicle.

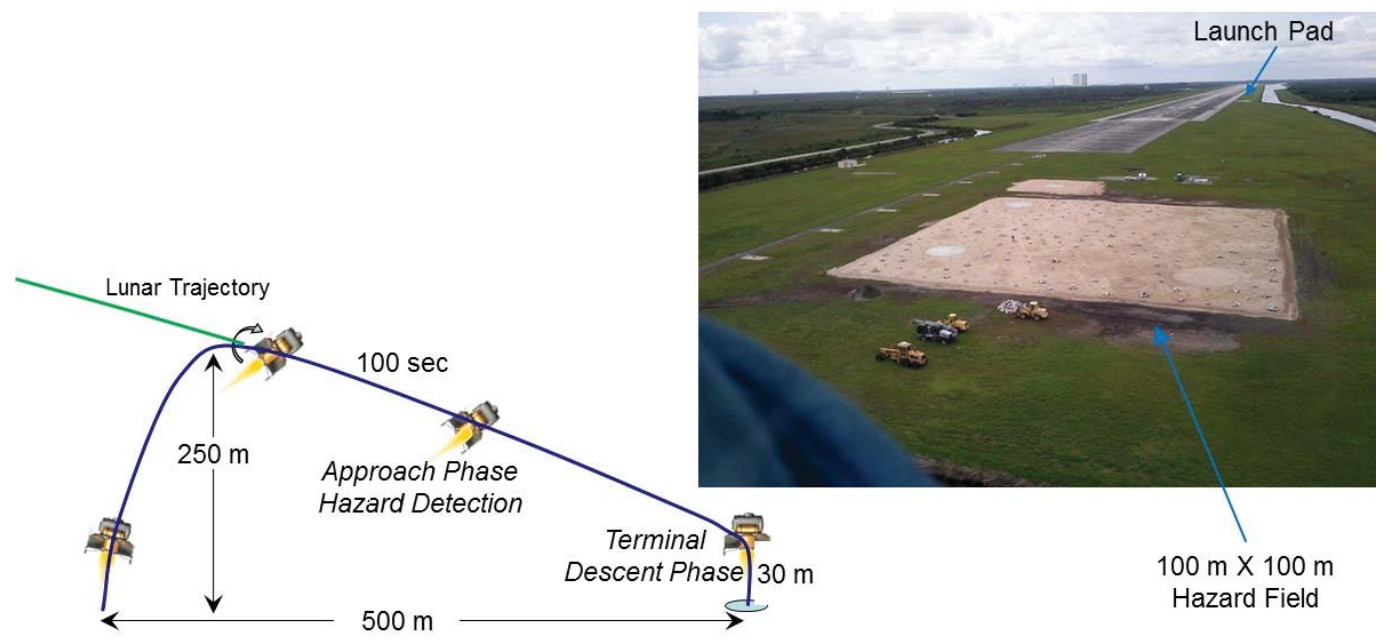

Figure 11. Flight profile for demonstrating autonomous safe landing at a hazard field (simulated lunar terrain) constructed at north end of Shuttle Landing Facility. 


\section{Sensor Systems Enhancements and Technology Maturation}

Thus far, the lidar sensors technology development has been focused on meeting the performance goals and ensuring reliable operation in field test campaigns. After successful closed-loop flight test, our focus is now shifting to optimizing the prototype systems designs and addressing space qualification requirements. The design optimizations will expand the operational envelop of the lidar sensors to satisfy the requirements of a wide range of missions to different destinations in the solar system. The design optimizations will also significantly reduce the size, weight, and power (SWaP) of the current prototype sensors. The design optimizations include removing excess engineering margins, eliminating unnecessary components monitoring devices, reducing the number of interconnects, and better thermal and packaging designs. The heat dissipation and thermal control subsystems account for a significant fraction of the size, mass, and power of the current systems. These design optimizations will result in over $40 \%$ SWaP reduction for the Doppler lidar and Laser Altimeter, and close to 70\% SWaP reduction for the Flash lidar.

It is also very beneficial to implement some incremental improvements in performance of these lidars to ensure their compliance in meeting all the requirements of future missions. The two noteworthy enhancements are: 1) increasing the Doppler lidar maximum velocity by a factor two; 2) increasing the number of Flash lidar image pixels by at least a factor of four. The maximum LOS (Line-Of-Sight) of the Doppler lidar is currently $70 \mathrm{~m} / \mathrm{sec}$, but it is necessary to increase it to about $140 \mathrm{~m} / \mathrm{sec}$ to comply with a wide range of descent trajectories being considered for the Moon and Mars landings. This will be achieved by increasing the bandwidth of the lidar's receiver and signal processor.

Increasing the number of Flash lidar image pixels can be achieved by employing a larger focal plane array with higher sensitivity that allows the use of a reasonable size transmitter laser. ALHAT project actively pursued the development of such focal plane array, but considerably more resources are needed to complete its development and implement it in current prototype system. Therefore, we developed a Super-Resolution (SR) technique that can significantly increase the effective number of image pixels ${ }^{13}$. We have already demonstrated a factor of four enhancement in image resolution at the NASA-LaRC Lidar Test Range ${ }^{13,14}$. SR is a well-established technique for enhancing 2-D images and over the years, a large number of algorithms have been developed for processing intensity images. SR algorithm takes advantage of sub-pixel shifts between multiple image frames of the same scene to produce a higher resolution image. We have extended the conventional 2-D SR technique to Flash lidar 3-D images. The SR algorithm also provides all six components of the vehicle's position and orientation vector that can drastically simplify the current HDS and HRN algorithms and ease the requirements of the lidar pointing gimbal.

The lidar sensors design optimizations and upgrades can be pursued in parallel with space qualification activities. Even though the development of the prototype lidar sensors were focused on functionality, the eventual goal of deployment in space was always in consideration. Throughout their developments, attempts were made to select components and subsystems with a path to space whenever possible. For example, the design of the data acquisition and signal processor architecture were based on components with either available space-grade equivalents or the promise of space-qualified versions. A study is currently underway aimed at developing space qualification plans for the major components of each of the lidar sensor systems. This study tries to leverage the heritage and capabilities established by past and current relevant missions such as STORRM, SpaceX Dragon Eye, OSIRIS-Rex, LRO, and LADEE.

\section{Conclusion}

A suite of three lidar sensor systems has been developed at NASA-LaRC under ALHAT project for enabling future NASA landing missions requiring hazard avoidance and precision navigation. These lidar sensors are a Doppler Lidar, a long distance Laser Altimeter, and a three-Dimensional Imaging Flash Lidar. These lidar sensors perform five essential landing functions: Altimetry, Velocimetry, Terrain Relative Navigation (TRN), Hazard Detection and Avoidance (HDA) and Hazard Relative Navigation (HRN). The capabilities of these advanced lidar sensors were evaluated through a series of tests at the NASA-LsRC's Lidar Test Range Facility and five helicopter and fix-wing aircraft flight tests during different phases of their development. These tests led to the development two sets of fullyautonomous prototype systems with integrtared real-time processors. One set of the prototype units were integrated into a rocket-powered free-flyer vehicle (Morpheus) along with the other components of the ALHAT landing system to demonstrate hazard detection and precision landing. In these flight tests, the ALHAT system used the Flash Lidar 3-D images to identify the terrain hazards and select a safe landing location. The ALHAT landing system then utilized the Doppler Lidar and Laser Altimeter data to navigate the vehicle and execute a landing maneuver at the selected safe location surrounded with hazardous rock piles and craters. The Morpheus closed-loop flight tests were a successful demonstration of ALHAT landing system and an excellent opportunity for validating the lidar sensors for 
future robotic and manned missions to solar system bodies. Based on results from Morpheus flight tests, a plan has been developed to continue the maturation of the ALHAT lidars technologies leading to space-qualified units. This plan includes a number of design upgraded and optimization that can further expand the operational envelop of the lidars and significantly reduce their size, mass, and power.

\section{Acknowledgments}

The authors are grateful to ALHAT project manager, Chirold Epp of NASA Johnson Space Center, for his guidance and support. We also would like to thank NASA's Advanced Exploration Systems (AES) program office for their continued support. The authors also acknowledge the ALHAT team members from NASA Johnson Space Center and NASA Jet Propulsion for their collaboration, and NASA Kennedy Space Center for facilitating the field tests.

\section{References}

1. Epp, C. D., Robinson, E. A., and Brady, T., “Autonomous Landing and Hazard Avoidance Technology (ALHAT)", Proc. of IEEE Aerospace Conference, paper no. 1644, 2008.

2. Huertas, A., Johnson, A. E, Werner, R. A., Maddock, R A., "Performance Evaluation of Hazard Detection and Avoidance Algorithms for Safe Lunar Landings,” Proc. IEEE Aerospace Conference, PP 1-20, 2010.

3. Brady, T, Paschall, S., and Crain, T.P., "GN\&C Development for Future Lunar Landing Missions," Proc. AIAA Guidance, Navigation, and Control Conf, 8444, 2010.

4. Project Morpheus Website http://morpheuslander.jsc.nasa.gov

5. Rutishauser, D., Epp, C. D., and Robertson, E. A., "Free-Flight Terrestrial Rocket Lander Demonstration for NASA's Autonomous Landing and Hazard Avoidance Technology (ALHAT) System," Proc. of AIAA SPACE 2012.

6. Stettner, R., Bailey, H., and Silverman, S., "Three Dimensional Flash Ladar Focal Planes and Time Dependent Imaging," International Symposium on Spectral Sensing Research, Bar Harbor, Maine, 2006.

7. Stettner, R., "Compact 3D Flash LIDAR video cameras and applications," Proc. of SPIE Vol. 7684, 768405, 2010.

8. Pierrottet, D. F., Amzajerdian, and F., Barnes, B.W., “A long-distance laser altimeter for terrain relative navigation and spacecraft landing," Proc. SPIE. 9080, 2014

9. Carson, J.M., Robertson, E.A., Pierrottet4, D.F., Roback, V.E., Trawny, N., Devolites, J.L., Hart, J.J., Estes, J.N., and Gaddis, J.S., "Preparation and Integration of ALHAT Precision Landing Technology for Morpheus Flight Testing," 4313, Proc AIAA Space 2014.

10. Carson, J.M., Bailey, E.S., Trawny, N., Johnson, A.E., Roback, V.E., Amzajerdian, F., and Werner, R.A., "Operations concept, hardware implementation and ground-test verification of a hazard detection system for autonomous and safe precision lunar landing,".AAS/AIAA Astrodynamics Specialist Conference, 2013.

11. Roback, V. E., Bulyshev, A., Amzajerdian, F., Brewster, P. F., Barnes, B. W., Kempton, K. S., and Reisse, R. E., "Helicopter Flight Test of 3-D Imaging Flash LIDAR Technology for Safe, Autonomous, and Precise Planetary Landing," Proc. of SPIE, Vol. 8731, SPIE Defense Security, and Sensing Conference, 2013.

12. Pierrottet, D. F., Amzajerdian, F., Petway, L. B., Barnes, B. W., Lockard, G., and Hines, G. D., "Navigation Doppler Lidar Sensor for Precision Altitude and Vector Velocity Measurements Flight Test Results." SPIE Defense and Security Symposium, Orlando, FL, (2011).

13. Bulyshev, A., Amzajerdian, F., Roback, V.E., Hines, G.D., Pierrottet, D.F., and Reisse, R., "Three-dimensional super-resolution: theory, modeling, and field test results," Applied Optics, Vol. 53, pp.2583-2594, (2014.

14. Bulyshev, A., Amzajerdian, F., Roback, V.E., and Reisse, R., "A super-resolution algorithm for enhancement of flash lidar data: flight test results," Proc. SPIE. 9020, 2014. 